\section{Captures}

Figures, théories et pratiques de l'imaginaire

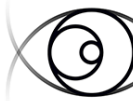

\section{Les images ont chaud!}

\section{La fièvre post-photographique au regard du surréalisme}

C A P $T$ T U R Figures, théories et pratiques de limaginaire revue interdisciplinaire

\title{
Ji-Yoon Han
}

Volume 1, Number 1, 2016

Post-photographie?

URI: https://id.erudit.org/iderudit/1059826ar

DOI: https://doi.org/10.7202/1059826ar

See table of contents

Publisher(s)

Figura, Centre de recherche sur le texte et l'imaginaire

ISSN

2371-1930 (digital)

Explore this journal

Cite this article

Han, J.-Y. (2016). Les images ont chaud! La fièvre post-photographique au regard du surréalisme. Captures, 1(1). https://doi.org/10.7202/1059826ar
Article abstract

L'article répond à la proposition commissariale de Joan Fontcuberta pour l'édition 2015 du Mois de la Photo à Montréal, placée sous les auspices de " la condition post-photographique ». Par des rapprochements entre les œuvres exposées et des exemples de l'histoire de la photographie - plus spécifiquement la photographie surréaliste -, il interroge cette condition post-photographique dont Fontcuberta postule qu'elle incarne « un nouvel ordre visuel » résultant d'« une inflation des images sans précédent ».
This document is protected by copyright law. Use of the services of Erudit (including reproduction) is subject to its terms and conditions, which can be viewed online.

https://apropos.erudit.org/en/users/policy-on-use/ 
Version enrichie de cet article : http://revuecaptures.org/node/227

\section{Les images ont chaud!}

\section{La fièvre post-photographique au regard du surréalisme}

\section{Ji-Yoon Han}

Résumé :

L'article répond à la proposition commissariale de Joan Fontcuberta pour l'édition 2015 du Mois de la Photo à Montréal, placée sous les auspices de " la condition post-photographique ». Par des rapprochements entre les œuvres exposées et des exemples de l'histoire de la photographie — plus spécifiquement la photographie surréaliste - il interroge cette condition post-photographique dont Fontcuberta postule qu'elle incarne « un nouvel ordre visuel » résultant d'« une inflation des images sans précédent ».

Le présent article répond à la proposition commissariale de Joan Fontcuberta pour l'édition 2015 de la biennale du Mois de la Photo à Montréal, placée sous les auspices de « la condition post-photographique ». Par un exercice de contrepoint historique, en effectuant des rapprochements entre les œuvres exposées et des exemples de l'histoire de la photographie — plus spécifiquement, de la photographie surréaliste —, il pose quelques questions élémentaires pour situer cette condition post-photographique dont Fontcuberta postule qu'elle incarne « un nouvel ordre visuel » résultant d'« une inflation des images sans précédent » (2015: 6) : quelle histoire de la photographie permet de penser la post-photographie? Dans quelle mesure cette histoire éclaire-t-elle les mutations et les permanences qui ont cours dans les pratiques post-photographiques de l'image? Comment, dans ce contexte, peut-on articuler le rapport historique entre le passé et le présent?

La description et l'analyse de trois cas précis de convergence entre les usages surréalistes de la photographie et la post-photographie telle qu'illustrée par le Mois de la Photo conduiront, dans un deuxième temps, à exposer quelques considérations d'ordre méthodologique sur les enjeux et les modalités de l'articulation entre le passé et le présent. L'article proposera ensuite une réflexion plus approfondie sur la crise de surabondance et de fièvre visuelle caractéristique de la post-photographie aujourd'hui, dans la mesure où elle correspond également au contexte dans lequel la photographie surréaliste s'est développée entre les deux guerres. Enfin, la question de l'articulation historique sera réinvestie, cette fois en inversant la flèche chronologique du temps, afin de mettre en lumière les processus rétrospectifs qui motivent l'écriture de l'histoire.

\section{Beau comme la rencontre fortuite...}

Plusieurs œuvres exposées dans ce Mois de la Photo 2015 présentaient des similarités troublantes non seulement avec le surréalisme, dont on peut dire qu'il a largement infiltré notre rapport aux images depuis la 
seconde moitié du $X X^{e}$ siècle, mais, plus spécifiquement, avec la photographie surréaliste des années 20 et 30. Ces correspondances s'observent d'abord sur un plan iconographique, c'est-à-dire de ressemblance visuelle entre les images; elles concernent également, de manière plus essentielle, les usages du médium photographique et le rapport à l'image dont ceux-ci témoignent. Prenons, presque au hasard de la déambulation parmi le foisonnement des propositions artistiques exposées dans le cadre de la biennale, trois cas de coïncidences flagrantes, belles - suivant la formule de Lautréamont reprise par les surréalistes comme l'une de leurs devises - « comme la rencontre fortuite sur une table de dissection d'une machine à coudre et d'un parapluie ».

Au centre Clark, l'installation en réseau Memories Centers (2014-2015) de Grégory Chatonsky et Dominique Sirois présentait des séquences oniriques aléatoires générées par un logiciel. Celui-ci associait, d'une part, des mots-clés extraits de la base de données DreamBank, compilant plus de vingt mille récits de rêves-', et d'autre part, des images correspondant sur Internet aux recherches associées à ces mêmes mots-clés. La médiation numérique (banque de données, logiciel, serveur Internet) permettait de donner forme, ainsi que le soulignait le texte explicatif accompagnant l'œuvre, au " fantasme d'une machine à projeter les rêves ", mais à l'inverse, elle fournissait également une métaphore onirique à nos recherches quotidiennes sur la toile : comme si l'activité de navigation sur Internet, couplant les mots et les images par algorithmes, faisait déborder le rêve dans la vie consciente.

Parmi les pratiques photographiques où les surréalistes ont excellé, le photomontage fut l'un des procédés privilégiés pour rendre compte de ce rapport entre les mondes de la veille et du rêve, puisant à même la masse de photographies nouvellement disponible, en particulier dans la presse illustrée, afin de recomposer des images tantôt soigneusement élaborées, tantôt confiées au hasard de l'automatisme. Ainsi Max Ernst avait-il conçu Rêves et hallucinations (1926), un montage associant des photographies et des réclames, toutes découpées dans des journaux, et qui proposait un séquençage d'images et de mots déjà existants, déjà donnés dans le réel, mais remixés en un emblème nouveau de l'activité onirique et hallucinatoire. Avec les moyens technologiques d'aujourd'hui, Chatonsky et Sirois ont mis au point un dispositif permettant de recomposer le visible et le lisible, dans la perspective de faire émerger, à l'instar d'Ernst, ce qu'on pourrait nommer avec Walter Benjamin un « inconscient optique 2 », c'est-à-dire un inconscient technique qui n'apparaît qu'à notre corps défendant, au-delà de toute recherche intentionnelle : par automatisme hier, par algorithme aujourd'hui.

Les collections de photographie vernaculaire présentées par Erik Kessels à la galerie Occurrence reprenaient quant à elles un ethos bien connu des surréalistes, soit la collection passionnée de petits objets de rien, rescapés des poubelles de l'histoire. Objets incongrus chinés dans les marchés aux puces, graffitis découverts 
au hasard d'une rue parisienne, passages désuets témoins d'une époque révolue, affiches ou photographies orphelines ayant perdu tout usage : les surréalistes se sont emparés des figures baudelairiennes du flâneur et du chiffonnier pour mettre en œuvre une anthropologie de la modernité, où le merveilleux se loge — et c'est leur contribution singulière - dans la poésie du banal et des images sans qualité. Paul Éluard a ainsi réuni une imposante collection de cartes postales 1900, qu'il désignait comme " la petite monnaie de l'art », en particulier des cartes pornographiques aux atmosphères sépia ou colorisées à l'aniline 3 .

L'installation All Yours (2015), spécifiquement réalisée pour la biennale montréalaise, recourait justement au format de la carte postale pour mettre en forme des séries de photographies trouvées, souvent triviales, telles que des images de tir photographique dans des fêtes foraines, les photos d'identité d'une même femme à travers le temps, ou encore des collections de portraits de sœurs jumelles. Les cartes, placées sur des tourniquets mobiles comme dans une boutique de souvenirs, provenaient de quelques albums déjà publiés par Kessels, et qui réunissaient de façon thématique des images anonymes, achetées pour la plupart dans les marchés aux puces. En extrayant ces images de la masse du visible pour les repositionner dans un nouveau contexte (d'album ou d'installation), Kessels dote celles-ci d'un sens et d'un intérêt qu'elles n'avaient pas jusque-là, et ce geste est comparable aux surréalistes qui réussissaient si bien à inquiéter la photographie documentaire la plus banale, par une décontextualisation et un positionnement dissonnant dans les pages d'une revue $\underline{4}$.

Enfin, le projet Tous Décavés d'Isabelle Le Minh, à la galerie SBC, présentait plusieurs installations photographiques inédites s'appropriant des techniques visuelles marquantes de l'histoire de la modernité : Scaring from the Square, After François Morellet(2015) reprenait ainsi la formule desRépartitions aléatoires de carrés pour les traduire en codes QR;Digitométrie, After Yves Klein (2015) revisitait les Anthropométries de l'époque bleue à travers le relevé des empreintes digitales laissées sur des écrans tactiles; Portraits soufflés, After Alphonse Bertillon (2015) compilait des photographies publiées par les usagers de Facebook, suivant la formule des anthropométries judiciaires, soit des séries d'organes isolés (le nez, l'oreille, la bouche, etc). Ces œuvres sont le produit d'un geste typiquement postmoderne d'appropriation de motifs extraits du passé en vue d'une actualisation dans le présent (ici un présent technologique).

Cependant, l'installation Portraits soufflés ne peut manquer de rappeler également un photomontage fameux réalisé par Salvador Dalí en 1933 et publié dans la revue d'art Minotaure, sous le titre Le phénomène de l'extase. Le peintre y reprenait les séquences d'oreilles d'un tableau synoptique de Bertillon afin de les transformer en fétiches, détournant ainsi la pulsion de contrôle policier par la photographie au profit d'une pulsion scopique visant la perte de tout contrôle - cette dernière étant figurée dans le photomontage par plusieurs gros plans de visages féminins, visiblement en extase. Nul doute que l'installation d'Isabelle Le Minh 
ne possède pas le dessein érotique de l'œuvre de Dalí. En revanche, elle opère un même geste de détournement, presque symétrique à celui de Dalí, où l'exhibition jubilatoire de soi sur le convivial « livre des visages " s'avère alimenter un puissant contrôle des identités faciales, à travers le mimétisme normatif des opérations visuelles qui y ont cours, ainsi que le branding du désir et des identités qui en découle.

Au-delà de ces rapprochements quelque peu cavaliers, deux observations supplémentaires permettront ici de donner une portée plus générale aux affinités entre la photographie surréaliste et la post-photographie. Tout d'abord, des penseurs tels que William J. Mitchell, Fred Ritchin, et Joan Fontcuberta, s'accordent à caractériser la post-photographie par une remise en question profonde de la véracité de l'image photographique, entraînant, d'une part, le développement des usages fictifs de la photographie, au détriment de la valeur testimoniale de celle-ci, et d'autre part, de nouveaux régimes de vérité de l'image. Or cette valeur fictive conférée à l'image photographique constitue l'un des apports principaux de la photographie surréaliste des années 20 et 30. L'historien de la photographie Herbert Molderings en a ainsi proposé un panorama, à travers des exemples de mise en scène photographique relevant d'une esthétique du «possible », tels que les portraits de Duchamp travesti en Rrose Sélavy, réalisés par Man Ray, les autoportraits performatifs de Claude Cahun, le photo-récit La Subversion des images de Paul Nougé, ou encore les saynètes amateures de Magritte.

Il est en outre remarquable que les artistes réunis au nom de la condition post-photographique produisent euxmêmes rarement des images, élaborant plutôt des pratiques de collecte, de tri et de mise en forme de cellesci 5 . L'on ne s'étonnera donc pas que Kessels soit à la fois publicitaire et éditeur, artiste et commissaire d'expositions, ou que Le Minh navigue également entre la position d'artiste et celle de commissaire, à l'instar de Fontcuberta lui-même. La surabondance des images et leur surdisponibilité y sont sans doute pour beaucoup. Ainsi que le suggère Suzanne Paquet dans son essai « Trafics numériques. Le web en cascades d'images (photographiques) », publié dans le catalogue du Mois de la Photo :

Chaque chose du monde - ou presque - a désormais son double, une image circulant dans un flux parallèle, dans un espace autre où [...] les images tiennent lieu de destinations et les parcours par elles induits s'apparentent généralement à la flânerie, ou à une sorte de dérive; des trajectoires aléatoires qui ne sont souvent guidées que par les préférences des autres. (150.)

Les circuits numériques de l'image rappellent ainsi, écrit encore Paquet, les pratiques situationnistes de la dérive et de la psychogéographie — non sans ironie à l'égard du pourfendeur de la société du spectacle. Or celles-ci procédaient elles-mêmes des déambulations urbaines des surréalistes, paysans de Paris privilégiant la trouvaille à la fabrication : la dérive contemporaine dans cet espace autre où tous les chemins mènent aux images s'apparente ainsi à la quête surréaliste d'images-étincelles à la faveur du hasard objectif. 


\section{Entre hier et aujourd'hui, ou comment articuler les coïncidences historiques}

Comment dès lors penser la correspondance entre la post-photographie et la photographie surréaliste? Faut-il faire de celle-ci le point d'origine de celle-là? Doit-on en déduire l'inexactitude du postulat selon lequel la postphotographie incarne une révolution sans précédent? Mais alors, que faire des transformations technologiques, pourtant indéniables, qui séparent le début de ce millénaire du siècle précédent? Ne compteraient-elles pour rien? Comment articuler les coïncidences entre les images dans la succession du temps? Le rapport entre post-photographie et photographie surréaliste ne semble pas devoir être ici d'ordre génétique. Établir une généalogie de la post-photographie qui en identifierait les origines, en établirait la préhistoire et les filiations, contribuerait certes à l'érudition, mais ne nous avancerait pas beaucoup dans l'analyse et la compréhension de notre rapport aux images. D'ailleurs, à chercher les « premières fois » des pratiques de détournement, de fictionnalisation et de remixage de la photographie, il faudrait remonter bien en amont des surréalistes, dans I'histoire de la photographie vernaculaire elle-même, et en particulier, ainsi que Clément Chéroux l'a analysé dans sa thèse de doctorat récemment publiée (2015), dans les pratiques de récréation photographique qui se développent dès la fin du XIXe siècle, au moment où les premiers appareils simples d'utilisation et de coût modique sont introduits sur le marché et rapidement popularisés auprès des amateurs.

Dans le cas présent, l'intérêt des analogies historiques serait plutôt attribuable à la notion de « postphotographie » elle-même, dans la mesure où celle-ci exige, par son préfixe, de penser le contemporain dans une position temporelle de l'après-coup. Cette position est désormais convenue, dans le contexte désabusé d'un postmodernisme pris dans la sempiternelle répétition du même ou dans un mouvement vertigineux de différences, noyant en tout cas la conscience historique dans d'interminablesafters. Mais on peut aussi l'envisager différemment, en un éclair, c'est-à-dire comme une de ces rencontres dialectiques de l'histoire entre l'Autrefois et le Maintenant qui, selon Walter Benjamin, sont productrices d'images :

Il ne faut pas dire que le passé éclaire le présent ou que le présent éclaire le passé. Une image, au contraire, est ce en quoi l'Autrefois rencontre le Maintenant dans un éclair pour former une constellation. En d'autres termes, l'image est la dialectique à l'arrêt. Car, tandis que la relation du présent avec le passé est purement temporelle, continue, la relation de l'Autrefois avec le Maintenant présent est dialectique : ce n'est pas quelque chose qui se déroule, mais une image saccadée. (478-479.)

Privilégier la constellation et l'étincelle, c'est donner une chance au " post » d'être non pas tant ce qui vient toujours trop tard, mais ce qui se rapporte au passé de manière discontinue, sur le mode du trait d'union et des raccourcis temporels, par saccades. Et c'est peut-être en cherchant moins des éclairages historiques que des secousses électriques que l'on pourra apporter quelque lumière sur notre rapport aux images. 
L'intérêt de la comparaison entre la post-photographie et la photographie surréaliste se fonderait ainsi plutôt sur le fait que celle-ci s'est développée, au cours des années d'entre-deux-guerres en Europe, dans un contexte que l'on qualifie couramment de « seconde naissance » de la photographie 6 . László Moholy-Nagy, apôtre de la Nouvelle Photographie en Allemagne, en a donné la formule en 1929 : « II y a cent ans que la photographie est inventée, elle vient seulement d'être vraiment découverte » (29). L'idée sera rapidement reprise et développée par les défenseurs de la photographie moderne, parmi lesquels Waldemar George, qui compose en 1930, dans «Photographie, vision du monde », une véritable ode à la modernité et même, selon I'historien Christian Bouqueret, le « manifeste international de la Nouvelle Vision » (50) :

L'avènement de la conscience photographique est un phénomène d'après-guerre. Ladécouverte de la photographie coïncide avec le centenaire de l'invention de Niepce [...]. II a fallu un siècle pour que les hommes saisissent la véritable portée d'un art ravalé au niveau d'une industrie vulgaire, sinon par le public, du moins par les élites. (George : 134.)

S'établit donc une distinction entre l'invention de la photographie et sa " découverte ", entre l'apparition d'une technique et l'avènement d'une conscience proprement photographique, débarrassée notamment du modèle pictural que les pictorialistes avaient fait peser sur le médium afin d'en justifier les usages artistiques. Et les surréalistes, sans avoir explicitement commenté cette découverte, y ont pleinement participé, à travers leurs expérimentations et leurs usages de la photographie.

La saccade dialectique se produirait ainsi dans le rapprochement entre un commencement et une fin qui ne correspondent pas à une évolution technique, mais coïncident plutôt avec une « conscience » induisant un rapport nouveau aux images. En effet, les discours sur la post-photographie s'entendent pour décrire une persistance de la photographie après la photographie, pointant ainsi une forme de survivance du médium audelà de l'obsolescence de la technique qui a permis d'édifier une histoire de la photographie. Joan Fontcuberta suggère ainsi :

Nous assistons non pas à la naissance d'une technique, mais à la transmutation de certaines valeurs fondamentales. Sa carcasse reste indemne; c'est son âme qui se transforme selon une sorte de métempsychose. Nous sommes témoins non pas de l'invention d'un procédé, mais de la désinvention d'une culture : le démantèlement de la visualité que la photographie a déployé de façon hégémonique durant un siècle et demi. (2015: 10.)

À l'instar de la seconde naissance de la photographie, il ne s'agit donc pas d'une vraie « mort » du médium, mais d'une mutation d'ordre anthropologique, presque métaphysique, à l'endroit des images et de leur «âme ». La pensée métaphorique vient alors au secours d'une transformation des consciences : les 
métaphores de la naissance et de la mort permettent ainsi de nommer ce qui n'a pas encore de nom; Fontcuberta pour sa part recourt abondamment aux métaphores bibliques et mythologiques pour penser la « révolution » de la post-photographie dans ses livres Le Baiser de Judas et Pandora's Camera.

\section{Surchauffes médiales : penser la fièvre des images}

"La post-photographie renvoie à la photographie qui déferle dans l'espace hybride de la sociabilité numérique, une conséquence de la surabondance visuelle » (Fontcuberta, 2015: 6) : la nouvelle ère visuelle est caractérisée par la sursaturation des images. Les œuvres exposées dans le cadre du Mois de la Photo en donnaient de multiples illustrations : outre celles commentées plus haut, toutes fondées sur d'abondantes collections d'images, Mémoires (2015) de Roberto Pellegrinuzzi consistait en une immense installation nébuleuse réunissant un quart de million de clichés pris avec un appareil numérique jusqu'à ce que le capteur en soit épuisé; l'm Google (2011-) de Dina Kelberman invitait le visiteur à dérouler sur une projection murale un fil continu d'images trouvées en ligne et ordonnées par affinités tantôt morphologiques, tantôt sémantiques; certaines œuvres vidéographiques enfin déployaient des diaporamas kaléidoscopiques frisant la stroboscopie - The World As Will and Representation, Archive 2007 (2007) de Roy Arden faisait défiler sur un unique écran près de trente mille images trouvées sur Internet, tandis que The Year of GIF (2013) et \#LLL, Looking, Listening, Looping (2014) de Paul Wong présentaient des mosaïques d'écrans mûs par des myriades d'images GIF animées.

La sursaturation désigne non seulement la massification des images, leur multiplication exponentielle et leur diffusion sans précédent grâce aux technologies numériques, mais elle sous-entend également un sentiment d'excès, de nausée et de pollution, provoqué par la quantité démesurée des images que nous produisons et consommons de manière compulsive, comme des boulimiques. En ce sens, la sursaturation correspond à une frénésie visuelle dont les effets présentent tous les symptômes d'une fièvre, soit un état pathologique qui porte atteinte à nos capacités physiques et mentales de percevoir et d'absorber les images. Fontcuberta affirme ainsi que

[n]ous vivons par conséquent une époque où les images sont abordées sous l'angle de l'excès, une époque où nous parlons davantage des conditions asphyxiantes de la production en masse que de son potentiel d'émancipation [...]. Les notions d'excès d'images, d'hypervisibilité et de voyeurisme universel — qui semblent aussi causer une forme d'aveuglement ou d'insensibilité —, cette apparente « épidémie des images ", méritent une analyse critique plus profonde. (2015: 12.)

L'économie post-photographique est certes quantitative, mais elle affecte puissamment les subjectivités, visiblement débordées par le flot des images. Si la masse d'images en circulation dans le monde aujourd'hui 
est effectivement sans précédent, le sentiment de fièvre et d'épidémie qui en découle n'est pas l'apanage de notre époque.

Précisément, une telle surchauffe médiale aura déjà eu lieu au moment de la seconde naissance de la photographie, qui coïncide avec l'émergence d'une culture visuelle fondée sur la profusion des images et leur diffusion massive. Cette culture, arrimée à tout un ensemble de mutations techniques, économiques, sociales et esthétiques, donne lieu à un véritable boom visuel qui traduit et comble, selon les témoignages de l'époque, une soif nouvelle d'images. Ainsi voit-on la production et la diffusion des journaux illustrés de photographies exploser : le magazine d'actualités $V u$ annonce, dès sa première livraison le 21 mars 1928, qu'il « mettra à la portée de l'œil la vie universelle », en publiant notamment « des pages bourrées de photographies » et « de sensationnels reportages illustrés » (anon: 11-12). Son rédacteur en chef Carlo Rim renchérit, dans un texte de « Défense et illustration de la photographie » : « La photographie est notre nouveau pain quotidien, un aliment sans cesse renouvelé offert à l'insatiable curiosité de nos yeux avides d'images », si bien, dit-il, que « notre culture est devenue visuelle $\gg(587)^{7}$.

L'historien de la photographie Olivier Lugon, dans un brillant article sur « la profusion des images dans la photographie allemande » entre 1925 et 1945, explique que l'art photographique, autrefois associé à la rareté, revendique désormais la quantité, dans le but de « renouveler de fond en comble la vision humaine en général » (91). Photogramme, photomontage, surimpression, instantané, gros plan, négatif, photographie abstraite ou document : tout concourt non plus à créer une œuvre au sens traditionnel, mais à donner du monde une « vision directe à saturation », selon la formule de Franz Roh, éditeur en 1929 de l'influent album Foto-Auge. 76 Fotos der Zeit. Si bien que dès 1929, le «photo-boom » dans les débats contemporains se renverse en "photo-inflation », en sentiments d'excès et de fatigue visuelle traduits dans le lexique de la crise financière, suscitant dès lors une pulsion sanitaire d'organisation de la masse des images : par la mise en série, la constitution du modèle de l'archive, et, après l'arrivée au pouvoir des national-socialistes, une pulsion de contrôle de la masse des images, aussi bien que de la masse par l'image.

Les surréalistes ont eux aussi répondu à cette nouvelle donne, en plaçant pour leur part l'ivresse de ce qu'Aragon appelait le stupéfiant image au cœur de leurs pratiques photographiques, c'est-à-dire en provoquant délibérément la déferlante des images, y cherchant une forme de dépossession hypnotique. Revenons à l'exemple dalinien $\underline{8}$. Dans les pages des numéros 3-4 de la revueMinotaure, paru en décembre 1933, le photomontage Le phénomène de l'extase était immédiatement précédé d'un long article du même Dalí intitulé «De la beauté comestible et terrifiante, de l'architecture Modern' Style ». Cet article richement illustré apportait une contribution essentielle, tant sur le plan visuel que discursif, à la méthode paranoïaque-critique élaborée par le peintre depuis la publication de La Femme visible en 1930. Conçue en réponse au modèle passif de 
l'automatisme et de l'hallucination propre au surréalisme des années 20, la méthode paranoïaque-critique vise, à travers l'instrumentalisation des délires interprétatifs qui caractérisent la paranoïa, à proposer un modèle actif de réalisation concrète des désirs. Parmi les images ainsi générées figure de manière privilégiée le jeu de l'image double, où une image en cache objectivement une autre, déclenchant un trouble perceptif en vue d'un accroissement des pouvoirs de la vision. Le développement de l'aptitude à une interprétation à la fois délirante et objective des images devait ainsi fournir un moteur essentiel au surréalisme des années 30 .

Dans l'article sur le Modern' Style, le phénomène de l'image double est provoqué par les légendes des illustrations photographiques strictement documentaires des architectures de Gaudí et de Guimard, fournies par Man Ray et Brassaï. Ainsi le gros plan d'un portail barcelonais est-il accompagné de l'invitation : « On pénètre dans les grottes par de tendres portes en foie de veau » (69). Le détail nocturne d'un ornement végétal du métro parisien se surprend même à parler : «Mange-moi! », tandis qu'un second réclame : « Moi aussi! » (75). Au-delà de la double image qui perturbe le regard, la verbalisation de l'image par les légendes entraîne une animation qui en exhibe la voracité — «Mange-moi! » signale tout autant la faim sexuelle de l'image que le désir exprimé par celle-ci d'être consommée, et donc de provoquer chez le regardeur un appétit de voir. De fait, la méthode paranoïaque-critique, d'abord articulée autour des motifs de la putréfaction et de l'excrément, s'oriente à partir de 1932 vers une thématisation de la comestibilité et du cannibalisme des images. Ainsi transformée en aliment, l'image est alors moins considérée dans sa dimension représentative (l'image double représente deux choses en une), que sous un aspect quantitatif : s'il veut assouvir sa faim, le délire paranoïaque-critique doit générer une prolifération d'images.

Cette pensée de la comestibilité peut être rapportée au «photo-boom » qui transforme les images en « notre nouveau pain quotidien », ainsi que Carlo Rim le formule au même moment (1932: 587) . Plutôt que de répondre à la profusion photographique par les moyens d'une hygiène visuelle, comme ce fut le cas dans le contexte allemand décrit par Lugon, Dalí procède à une littéralisation de la consommation des images, en en exaltant les vertus comestibles. De la sorte, il s'intéresse davantage à une perception intensifiée des images qu'à leur production : si lui-même appelle et provoque l'avalanche visuelle, c'est surtout en vue d'interroger la manière dont on les regarde et dont on les consomme. C'est précisément le propos de la monumentale installation de Wang Du, International Kebab (2008), qui constitue une proposition artistique particulièrement éloquente sur la sursaturation des images dans le monde contemporain : au centre d'une pièce tapissée de photographies jusqu'aux murs de manière à tracer la silhouette d'une skyline imaginaire, s'élève une broche tournante de photographies empilées à une hauteur de neuf mètres, et pesant sept tonnes; le visiteur est invité à monter sur un échafaudage construit autour de la broche d'images et à en découper des fragments, comme un véritable kebab, à l'aide de couteaux à pain mis à sa disposition 10 . Dans un parallèle saisissant entre l'abondance excessive des images et la surconsommation de la malbouffe à l'échelle globale, 
l'œuvre fusionne la viande et l'image, réduisant cette dernière à une matière comestible (quoiqu'indigeste), au détriment de son contenu propre.

Dans ce contexte, le photomontage Le phénomène de l'extase, par effet de contamination avec la mise en scène des images Modern' Style qui le précèdent, apparaît comme le point d'orgue de la comestibilité et de la voracité des images : l'extase. Celle-ci est traduite dans un montage particulièrement touffu qui offre la particularité de résulter, à la différence des photomontages dada et surréalistes généralement créateurs d'une réalité nouvelle (aussi irréelle qu'elle soit), en une kyrielle de petites images pulvérisées sur un fond noir. La répétition obsessive des motifs (têtes pâmées, yeux révulsés, oreilles découpées) a pour effet de réduire chaque fragment visuel à un signe dont le référent est double : la jouissance sexuelle et l'atomisation visuelle, articulées de manière à produire le tableau d'une image mangée - mastiquée, déchiquetée en petits morceaux, consommée. Ce faisant, Dalí réalise un montage qui entre en parfaite résonance avec les analyses de la post-photographie proposées par Fontcuberta : « photographs are no longer taken to preserve a memory, or to be kept. They are more like exclamations of vitality, extensions of our experiences » (2014: 2728). Quoi de plus exclamatif, quoi de plus vivant, qu'une image qui jouit?

\section{À partir d'aujourd'hui... ou l'histoire de la photographie écrite à rebours ${ }^{11}$}

Pour conclure cette première esquisse des coïncidences et saccades entre les images de la photographie surréaliste et celles de la post-photographie, retournons aux modalités de l'articulation historique. Les réflexions actuelles sur la notion de post-photographie doivent être inscrites dans un contexte plus large où la seconde révolution numérique suscite une interrogation nouvelle - un brin frénétique - sur l'ontologie de la photographie. Des institutions muséales majeures ont organisé dans les deux dernières années des expositions réinvestissant la définition de la photographie, sous des titres sans équivoque : Qu'est-ce que la photographie? (2015) demandait-on au Centre Pompidou, à la suite de l'exposition What Is A Photograph? (2014) organisée à l'International Center of Photography de New York, tandis que l'Art Institute de Chicago proposait Photography is... (2014-2015). L'influente revue critique Texte zur Kunst a quant à elle consacré son numéro de septembre 2015 au thème Photography. Si l'on peut débattre sur la terminologie, à savoir si nous sommes entrés dans une « condition post-photographique » ou s'il faut plutôt élargir la définition et le sens de la « photographie », il est clair que l'image photographique bénéficie aujourd'hui d'un moment singulier de ferveur théorique 12 .

La formule de la « condition post-photographique » invite, par sa référence à Lyotard, à considérer la fin des « grands récits » qui ont forgé l'histoire de la photographie en tant qu'outil privilégié du savoir. L'on observe en effet dans les débats actuels une mise en cause de l'indicialité du médium, donc de la valeur testimoniale qui a fondé, par exemple, les usages scientifiques et journalistiques de la photographie. À l'instar du 
postmodernisme également, l'idée d'une condition post-photographique produit une relecture du passé, d'une manière qui n'est pas nécessairement mélancolique. Rosalind Krauss et les auteurs associés à la revue October ont ainsi défendu, dans les années 80 et 90 , un postmodernisme critique qui ne se résume pas au pluralisme et au relativisme. Et c'est dans le cadre de ses réflexions sur l'art de son temps que Krauss a contribué à révéler un champ jusque-là largement occulté par l'histoire de l'art, à savoir la photographie surréaliste, allant ensuite jusqu'à proposer une relecture de la modernité au $X X^{e}$ siècle à l'aune de cette « part maudite » fraîchement exhumée de l'oubli (Krauss, 1985; 1996).

La photographie surréaliste a ainsi été " découverte ", c'est-à-dire qu'elle est entrée comme telle dans la conscience collective, il y a une trentaine d'années. Bien qu'elle ne soit pas passée inaperçue auprès de ses contemporains, comme en attestent brillamment les essais de Walter Benjamin dès 1930, elle n'a en effet jamais été pensée ni revendiquée comme une pratique singulière par les surréalistes, à la différence de la peinture, des objets, de la poésie. Et il est frappant que cette naissance retardée de la photographie surréaliste dans les discours critiques ait été contemporaine de l'éclatement du « grand récit » du modernisme. Mais allons plus loin : est-ce un hasard si l'intérêt spécifique pour les usages surréalistes de la photographie, à travers non seulement la manipulation en chambre noire, mais aussi et surtout la photographie récréative et vernaculaire, les collections de cartes postales, les détournements de documents, bref, tous ces usages qui ont permis ici d'établir des ponts entre la post-photographie et la photographie surréaliste - est-ce un hasard si ces pratiques ont été mises en lumière dans la dernière décennie, grâce aux travaux de Michel Poivert (2006) et à l'exposition de référence La Subversion des images organisée par le Centre Pompidou (2009)? Peut-être est-ce précisément à cause de l'émergence d'une condition post-photographique que le passé nous apparaît sous une nouvelle lumière, et qu'on peut réécrire, rétrospectivement, l'histoire de la photographie. Compte tenu de la photographie surréaliste.

1. La base de données DreamBank, réalisée par Adam Schneider et G. William Domhoff, du département de psychologie de I'Université de Californie à Santa Cruz, est accessible en ligne : www.dreambank.net.

2. Benjamin élabore cette notion dans sa « Petite histoire de la photographie » (1931) et dans « L'œuvre d'art à l'époque de sa reproduction mécanisée 》 (1936).

3. Une sélection de plus de cent cartes postales appartenant à Paul Éluard est publiée dans la luxueuse revue d'art Minotaure en décembre 1933 (nos 3-4). Sur les collections de cartes postales, je renvoie également à Chéroux et Eskilden (2007).

4. Parmi les nombreuses études sur les usages surréalistes de la photographie documentaire, je renvoie en particulier à la thèse publiée de lan Walker (2002), aux travaux de Michel Poivert (2006) et de Clément Chéroux (2010).

5. Dans un article récent, Clément Chéroux a souligné l'émergence d'une nouvelle génération de « non-photographes » qui ont, dans le contexte de l'« infobésité » caractéristique de la dernière décennie, « ce don rare d'être en mesure d'apprivoiser le flux des images » (2014: 106).

6. Cette appellation, fréquemment utilisée par les historiens de la photographie, n'a toutefois pas encore fait l'objet d'une conceptualisation précise.

7. Pour des analyses détaillées de cet essor de la photographie dans la presse illustrée, je renvoie aux travaux de Thierry Gervais (2014) et Vincent Lavoie (2010).

8. Ce passage reprend très partiellement les analyses d'une thèse de doctorat en cours de rédaction au département d'histoire de 
l'art et d'études cinématographiques de l'Université de Montréal, intitulée La métaphore vacante. Des dispositifs pulsionnels de l'image photographique (1929-1936).

9. Le pain - et plus spécifiquement la baguette de pain, associée à l'érection - constitue d'ailleurs l'un des motifs principaux des tableaux que Dalí réalise en 1932, tels que : Femme avec pain catalan; Pain français moyen avec deux œufs sur le plat sans plat, à cheval, essayant de sodomiser une mie de pain portugais; Pain anthropomorphe; Pain catalan, Pain anthropomorphe.

10. L'œuvre est présentée pour la première fois en 2008 , à l'occasion de la première exposition personnelle de l'artiste en Chine, à la galerie Tang Contemporary Art (Beijing). Elle a également été exposée lors de la seconde édition de La Force de l'Art à Paris en 2009.

11. À partir d'aujourd'hui... Reconsidering Postphotography. An international conference on the state of photography in global and digital times était le titre du colloque du Mois de la Photo à Montréal 2015, lors duquel une première version du présent texte a été présentée.

12. En témoignait tout particulièrement le colloque organisé au printemps 2015 en marge de l'exposition du Centre Pompidou, intitulé Où en sont les théories de la photographie?. Les interventions, notamment de Clément Chéroux, Johanne Lamoureux et André Gunthert, faisaient état de l'effervescence animant le « revival » théorique actuel autour de la photographie.

\section{Bibliographie}

[s. a.]. 1928. « Vu ». Vu, no 1, 21 mars, p. 11-12.

BenJamin, Walter. 2006. Paris, capitale du XIXe siècle. Le Livre des passages. Paris : Cerf, "Passages », $976 \mathrm{p}$.

Bovqueret, Christian. 1997. Des Années folles aux années noires. La Nouvelle Vision photographique en France 1920-1940. Paris : Marval, $285 \mathrm{p}$.

CLÉMENT, Chéroux. 2010. «L'image comme point d'interrogation ou la valeur d'extase du document surréaliste », dans Jean-Pierre CRIQUI (dir.), L'image-document. Entre réalité et fiction. Paris : Le BAL, «Les carnets du BAL », t. 1, p. 26-47.

CHÉroux, Clément. 2014. «Face au flux. Art contemporain et photographie vernaculaire à l'époque d'internet ". Artpress, vol. 2, no 34, aout-septembre-octobre, p. 103-107.

CHÉroux, Clément. 2015. Avant l'avant-garde. Du jeu en photographie 1890-1940. Paris : Textuel, 288 p.

CHÉROux, Clément et Ute ESKILDSEN. 2007. La photographie timbrée. l'inventivité visuelle de la carte postale photographique. Göttingen : Steidl, 215 p.

DALI, Salvador. 1933. «De la beauté terrifiante et comestible. De l'architecture Modern' Style». Minotaure, no 3-4, décembre, p. 69-76.

Fontcuberta, Joan. 2005. Le Baiser de Judas. Photographie et vérité, traduit de l'espagnol par Claude Bleton, Jacqueline Gerday et Claude de Frayssinet. Arles : Actes Sud, 222 p.

FontcuberTA, Joan. 2014. Pandora's Camera.Photogr@phy(link sends e-mail) After Photography, traduit de l'espagnol par Graham Thomson. Londres : Mack, 188 p.

FontCuBerTA, Joan. 2015. La Condition post-photographique. Montréal : Le mois de la photo à Montréal, $232 \mathrm{p}$.

GeORge, Waldemar. 1930. «Photographie vision du monde», dans Photographie 1930. Paris : Arts et 
Métiers Graphiques, p. 5-161.

Gervals, Thierry et Gaëlle Morel. 2014. La Fabrique de l'information visuelle. Photographies et magazines

d’actualité. Paris : Textuel, «L'écriture photographique », 239 p.

Krauss, Rosalind. 1985. Explosante Fixe. Photographie et Surréalisme. Paris : Centre Georges Pompidou, $243 \mathrm{p}$.

Krauss, Rosalind et Yve-Alain BoIs. 1996. L'Informe. Mode d'emploi. Paris : Centre Georges Pompidou, $251 \mathrm{p}$.

LAVole, Vincent. 2010. Photojournalismes, Revoir les canons de la presse Paris : Hazan, 239 p.

LugON, Olivier. 1994. “Photo-inflation”. La profusion des images dans la photographie allemande, 1925-

1945 ». Cahiers du Musée National d'Art Moderne, vol. 49, automne, p. 90-113.

MitcheLL, William John Thoma. 1994. The Reconfigured Eye. Cambridge : MIT Press, 283 p.

MoHoly-NAGY, László. 1929. «La photographie. Ce qu’elle était, ce qu’elle devra être ». Cahiers d’Art, no 1, p. 29.

Molderings, Herbert. 2009. L'Évidence du possible. Photographie moderne et surréalisme. Paris : Textuel,

"L'écriture photographique », $192 \mathrm{p}$.

POIVERT, Michel. 2006. L’image au service de la révolution. Photographie, surréalisme, politique

Cherbourg : Point du jour, $124 \mathrm{p}$.

Rıм, Carlo. 1932. «Défense et illustration de la photographie». Vu, no 214, 20, avril , p. 587.

RitchIN, Fred. 2009. After Photography. Londres : W. W. Norton \& Company, 199 p.

WALKER, Ian. 2002. City Gorged with Dreams. Surrealism and Documentary Photography in Interwar

Paris. Manchester : Manchester University Press, 228 p. 